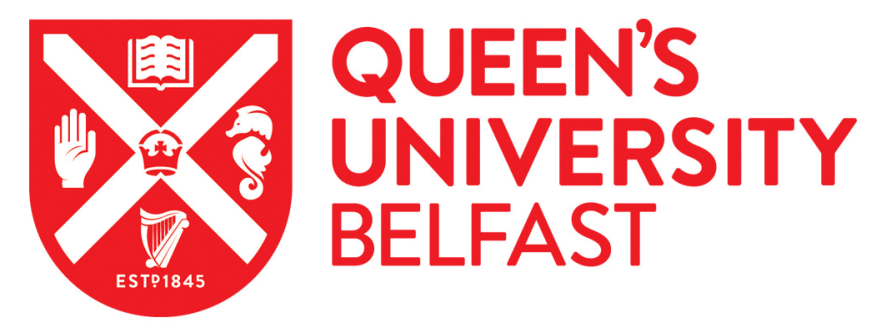

\title{
Compton profiles of Si: Pseudopotential calculation and reconstruction effects
}

Delaney, P., Kralik, B., \& Louie, S. G. (1998). Compton profiles of Si: Pseudopotential calculation and reconstruction effects. Physical Review B (Condensed Matter), 58(8), 4320-4324.

https://doi.org/10.1103/PhysRevB.58.4320

Published in:

Physical Review B (Condensed Matter)

Queen's University Belfast - Research Portal:

Link to publication record in Queen's University Belfast Research Portal

\section{General rights}

Copyright for the publications made accessible via the Queen's University Belfast Research Portal is retained by the author(s) and / or other copyright owners and it is a condition of accessing these publications that users recognise and abide by the legal requirements associated with these rights.

Take down policy

The Research Portal is Queen's institutional repository that provides access to Queen's research output. Every effort has been made to ensure that content in the Research Portal does not infringe any person's rights, or applicable UK laws. If you discover content in the Research Portal that you believe breaches copyright or violates any law, please contact openaccess@qub.ac.uk. 


\title{
Compton profiles of Si: Pseudopotential calculation and reconstruction effects
}

\author{
Paul Delaney, Balázs Králik, and Steven G. Louie \\ Department of Physics, University of California at Berkeley, Berkeley, California 94720 \\ and Materials Sciences Division, Lawrence Berkeley National Laboratory, Berkeley, California 94720
}

(Received 14 May 1997; revised manuscript received 3 February 1998)

\begin{abstract}
Using an ab initio pseudopotential calculation, we compute Compton profiles of silicon along the (100), (110), and (111) directions, and then reconstruct the pseudo-wave-functions to regain the oscillatory behavior of the all-electron valence wave functions near the atomic cores. We study the effect that this reconstruction has on the Compton profiles and their anisotropies. We find a decrease in the magnitude of the profiles at small wave vectors and in their anisotropies. These changes bring the theoretical predictions closer to experimental results. [S0163-1829(98)03628-5]
\end{abstract}

\section{INTRODUCTION}

Compton profiles ${ }^{1}$ study the energy profiles of photons that have been Compton scattered from electrons in condensed-matter systems. These profiles give information about the ground-state momentum density of the system, and can serve as a good check on the quality of the wave functions used in theoretical calculations. The differences or anisotropies between Compton profiles along different crystallographic directions are also investigated as they bring out some features hidden in the bell-like profiles. The accuracy with which the energy of the scattered photons can be measured has improved greatly in recent years, and so it is interesting to see if theoretical calculations of the Compton profiles match the more accurate experimental measurements. Many different condensed-matter systems are being studied, but silicon is being focused upon as a test case. ${ }^{2}$

Most older calculations of the Compton profiles of solids had used either tight-binding methods ${ }^{3}$ or empirical pseudopotentials ${ }^{4}$ to calculate wave functions, form momentum densities, and compute profiles. Both methods have disadvantages: the tight-binding method has parameters that must be chosen, and will not reproduce the wave functions well in interstitial regions, while the pseudo-wave-functions from the empirical pseudopotential calculation have lost the oscillatory behavior that the all-electron wave functions have near the atomic cores. Nevertheless, both methods can predict the profile shape well qualitatively, though it would seem from comparison with experimental data that theoretical calculations tend to overestimate the height of the profiles at low momenta and the size of the anisotropy in the profiles. ${ }^{5}$ In recent years, self-consistent calculations have been performed using the Korringa-Kohn-Rostoker (KKR) band-structure scheme $e^{6,7}$ and the full-potential linearized augmented-plane-wave method (FLAPW), ${ }^{8}$ and have compared favorably to recent experiments.

The first part of our work in this paper is to do an ab initio pseudopotential calculation on silicon, using densityfunctional theory ${ }^{9}$ in the local-density approximation, ${ }^{10}$ and to use the resulting valence pseudo-wave-functions to calculate the momentum density $n(\mathbf{p})$ and then the Compton profiles $J(q)$ along some high-symmetry directions. Then we reconstruct the all-electron valence wave functions from the pseudo-wave-functions by using a scheme devised by Meyer, Hummler, Elsässer, and Fähnle. ${ }^{11}$ By this reconstruction, we regain the oscillations in the core regions that were lost in using a pseudopotential in the initial calculation. With the reconstructed wave functions, we again calculate $n(\mathbf{p})$ and the various $J(q)$, in order to see if the addition of the core behavior of the wave functions causes any changes in the profiles or their anisotropies.

\section{DEFINITIONS}

The Compton profile $J(q)$ along a particular direction is what is directly measured in an experiment. By changing the orientation of the sample in an experiment, different crystallographic directions can be examined. Within the impulse approximation, ${ }^{1}$ we can calculate the profile along a direction given by the unit vector $\hat{e}$ from the formula

$$
J(q)=\int_{\text {all space }} d \mathbf{p} n(\mathbf{p}) \delta(\mathbf{p} \cdot \hat{\mathbf{e}}-q) .
$$

In other words, for a fixed value of $q$, we integrate $n(\mathbf{p})$ over the plane perpendicular to $\hat{e}$, which cuts the line along $\hat{e}$ at a signed distance $q$ from the origin.

Here, $n(\mathbf{p})$ is the probability density of the momentum of the electrons. In theoretical calculations these may either be just the valence electrons or just the core electrons, or both, while in experimental measurements on silicon both core and valence electrons scatter the photons and so contribute to the profiles. As the expression for the Compton profile is linear in the momentum density, a profile from just the core electrons can be calculated and then subtracted off from the total experimental Compton profile to obtain the valence profile. If we assume that the core electrons are unaffected by the presence of the other atoms in the crystal, then the momentum density of the core electrons will be spherically symmetric, so that the core Compton profiles will be the same along any direction, and will cancel out in the anisotropies. In our calculation, we calculate the momentum density and Compton profiles of the valence electrons only.

We define the valence momentum density $n(\mathbf{p})$ in such a way that it obeys the normalization 


$$
\int_{\text {all space }} d \mathbf{p} n(\mathbf{p})=4
$$

since silicon has four valence electrons per atom. From this it follows that the Compton profile $J(q)$ along any direction obeys the normalization

$$
\int_{-\infty}^{+\infty} d q J(q)=4
$$

\section{PSEUDOPOTENTIAL CALCULATION}

Using density-functional theory in the local-density approximation, we performed an $a b$ initio pseudopotential calculation on silicon in the diamond structure. We used a norm-conserving Hamann-Schlüter-Chiang pseudopotential, $^{12}$ with cutoff radii of 1.2 a.u., 1.3 a.u., and 1.7 a.u. for the $3 s, 3 p$, and $3 d$ states. In the generation of the pseudopotential, the $3 s$ orbital was fully occupied with two electrons, the $3 p$ orbitals with 1.6 electrons, and the $3 d$ orbitals with 0.4 electrons. A partial core correction was used for the exchange potential. ${ }^{13}$

The pseudo-wave-functions were expanded in a planewave basis set with an energy cutoff of $15 \mathrm{Ry}$, at the experimental primitive cell volume of 270.107 a.u. This corresponds to an expansion into approximately 265 plane waves. The Ceperley-Alder electron gas results ${ }^{14}$ as parametrized by Perdew and Zunger ${ }^{15}$ were used for the exchange-correlation potential. For $k$-space integrations, we used a Monkhorst-Pack ${ }^{16}$ unshifted $30 \times 30 \times 30$ grid of $k$ points in the primitive cell of the reciprocal lattice. We calculated the band energies for the lowest eight bands and the pseudowave-functions for the four occupied bands self-consistently on the 752 irreducible $k$ points. This density of $k$ points gave an effective distance between $k$ points of 0.033 a.u. This dense grid of $k$ points was chosen so that a linear interpolation scheme could be used to calculate the momentum density at other points in reciprocal space. Then the momentum density $n(\mathbf{p})$ was calculated from the pseudo-wave-functions by unfolding the data from the irreducible $k$ points into the full Brillouin zone, and then squaring the Fourier coefficients.

The Compton profiles along the cubic (100), (110), and (111) directions were calculated by integration of the momentum density using the linear tetrahedron method. ${ }^{17,18}$ For each profile, we performed 451 planar integrations of the momentum density, at momentum values between $q=0$ a.u. and $q=3.0$ a.u. Negative $q$ were not used as the profiles are symmetrical about $q=0$. Integration of each profile from -3.0 to +3.0 a.u. gave the expected value of 4 .

We checked the convergence of the calculation in the energy cutoff by doing a calculation with a $20 \times 20 \times 20$ grid at both $10 \mathrm{Ry}$ and $20 \mathrm{Ry}$. The convergence of the $k$-point grid was checked by running with an energy cutoff of $10 \mathrm{Ry}$, and two grid sizes of $20 \times 20 \times 20$ and $30 \times 30 \times 30$. In both cases, the profiles changed by at most $0.3 \%$ of the profile height at $q=0$.

\section{RECONSTRUCTION OF THE ALL-ELECTRON VALENCE WAVE FUNCTIONS}

The pseudo-wave-functions are constructed so as to have a smooth behavior near to the atomic cores, and so they lack some of the high-momentum Fourier components that the all-electron valence wave functions have. This implies that the momentum density $n$ (p) formed from the pseudo-wavefunctions will be reduced in weight at high momenta and, by normalization, have too much weight at low momenta. The same tendency will be found in the Compton profiles, although in the anisotropies it may be reduced if the corrections are spherically symmetrical.

To study this effect, we reconstruct the all-electron valence wave functions $\psi_{n \mathbf{k}}$ from the pseudo-wave-functions $\widetilde{\psi}_{n \mathbf{k}}$ using a scheme devised by Meyer, Hummler, Elsässer, and Fähnle. ${ }^{11}$ As the pseudopotential is constructed so as to give a pseudo-wave-function that reproduces the all-electron wave function outside some cutoff radius, we only have to change the pseudo-wave-functions inside a reconstruction sphere around the atoms, which includes this cutoff radius, and which should also enclose all of the core charge of the atom. Once the radius $r_{r e c}$ of this reconstruction sphere is chosen, we re-solve the Kohn-Sham equation using an allelectron potential for each valence wave function in the interior of this sphere. The necessary boundary conditions are specified by using the values of the corresponding pseudowave-function on the surface of the sphere. Once all of the pseudo-wave-functions have been reconstructed, we recalculate the all-electron potential and iterate to self-consistency.

In the calculation, we choose the reconstruction radius to be $r_{\text {rec }}=1.5$ a.u. At this radius, the $s, p$, and $d$ atomic pseudo-wave-functions and the atomic all-electron wave functions are very close to one another, so the use of the crystal pseudo-wave-functions as boundary conditions on the solutions inside the reconstruction sphere is justified. Using these reconstructed wave functions, we recalculate the momentum density. Again, we integrate the momentum density to find the Compton profiles along the three chosen cubic directions.

\section{RESULTS AND DISCUSSION}

In Fig. 1, we plot the valence momentum density along the (100) direction for both the pseudo-wave-functions and the reconstructed wave functions. By way of comparison, note that for a free Fermi gas with the same electron density, the momentum density would be a spherically symmetric step function, with the step located at the Fermi momentum $k_{F}=0.957$ a.u. and having a height of 1.09 in the present units. As expected, we see that adding in the oscillations of the valence wave functions in the core regions gives us a noticeable reduction in the momentum density at $q=0$. This lost weight should transfer outwards to higher momenta, and indeed we find that the reconstructed momentum density has a slowly decaying tail. This tail is small in magnitude, but extends far enough out in momentum space to make up for the loss in weight near the origin.

In Figs. 2, 3, and 4, we show the valence Compton profiles along the (100), (110), and (111) directions for the pseudopotential and reconstructed momentum densities, and 


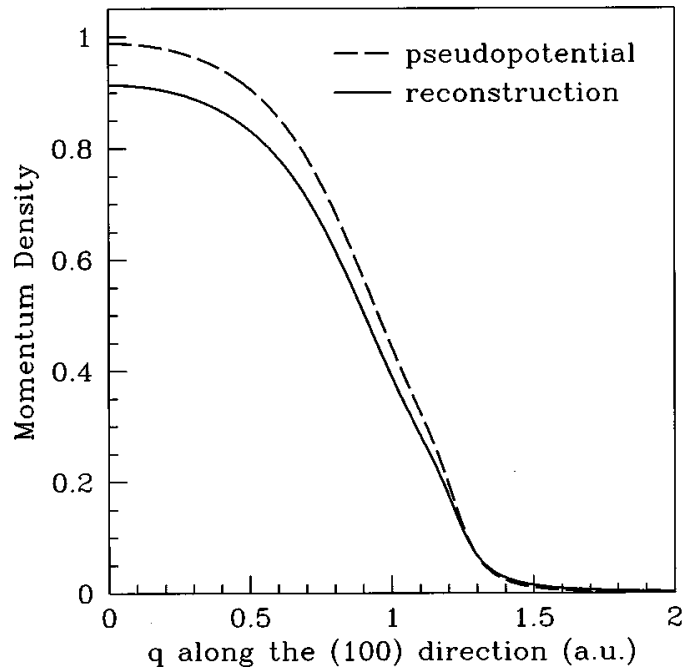

FIG. 1. Pseudopotential and reconstructed momentum density along the (100) direction, in units of electrons per atom per (atomic unit) ${ }^{3}$.

compare them with some experimental profiles. The two experiments we compare our profiles with are x-ray Compton profiles with incident photon energies of $29.5 \mathrm{keV}$ (Ref. 5) and $59.38 \mathrm{keV},{ }^{19}$ respectively. The $29.5-\mathrm{keV}$ experiment measured the (100) and (110) profiles, while the 59.38-keV experiment measured these profiles and the (111). In the figures, we display our theoretical results convoluted with the respective experimental resolutions of 0.084 and 0.12 a.u. So, on the left portion (negative $q$ values) of the (100) and (110) graphs we show the comparison with the $29.5 \mathrm{keV}$ experiment, and on the right portion (positive $q$ values) we compare with the 59.38 measurement. For the (111) direc-

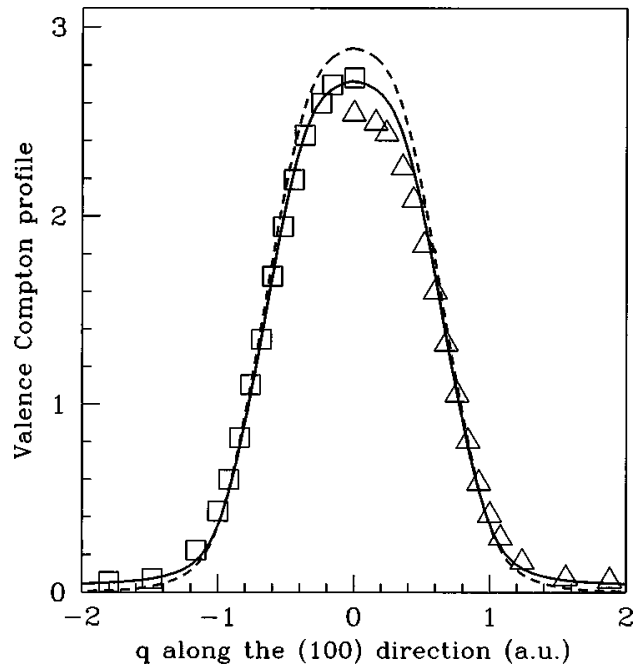

FIG. 2. Comparison between pseudopotential (dashed line) and reconstructed (solid line) Compton profiles along the (100) direction, and experimental data from the $29.5-\mathrm{keV}$ (Ref. 5) and 59.38$\mathrm{keV}$ (Ref. 19) experiments. On the left portion the $29.5-\mathrm{keV}$ experiment is graphed, with data points indicated by squares. On the right portion are the $59.38-\mathrm{keV}$ data, denoted by triangles. On each side of the graph, the theoretical profiles are convoluted with the appropriate experimental resolution function. The Compton profiles are in units of electrons per atom per atomic unit.

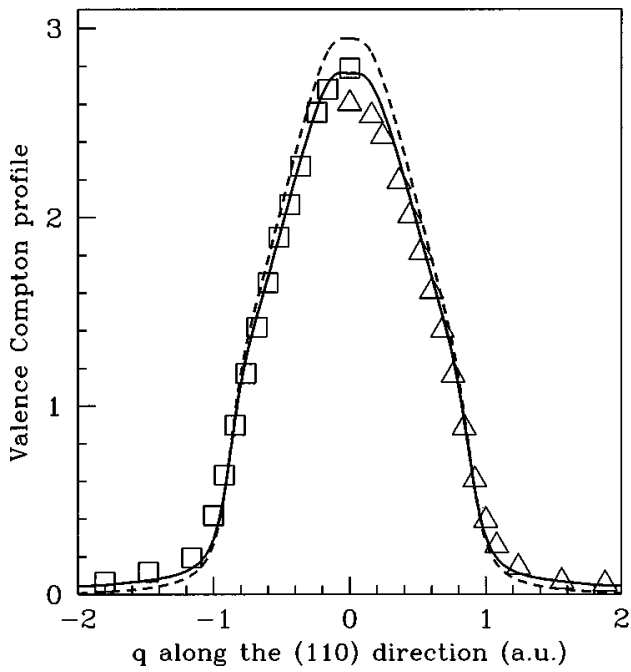

FIG. 3. Comparison between pseudopotential (dashed line) and reconstructed (solid line) Compton profiles along the (110) direction, and experimental data from the $29.5 \mathrm{-keV}$ (Ref. 5) and 59.38$\mathrm{keV}$ (Ref. 19) experiments. On the left portion the $29.5-\mathrm{keV}$ experiment is graphed, with data points indicated by squares. On the right portion are the $59.38-\mathrm{keV}$ data, denoted by triangles. On each side of the graph, the theoretical profiles are convoluted with the appropriate experimental resolution function. The Compton profiles are in units of electrons per atom per atomic unit.

tion, we have only the 59.38-keV data to compare with.

We see a substantial change in the valence Compton profiles due to the reconstruction procedure. The largest change is near the origin, where there is a reduction in the peak height of approximately $6 \%$. A long-range tail now appears, which compensates for the missing weight in the profile near zero. This tail is also visible in the momentum density in Fig. 1 , but due to the planar integration over the momentum den-

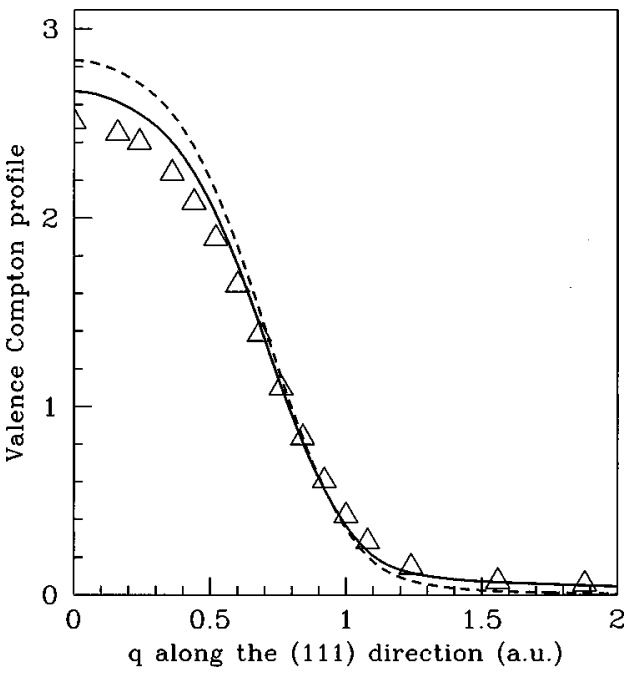

FIG. 4. Comparison between pseudopotential (dashed line) and reconstructed (solid line) Compton profiles along the (111) direction, and experimental data from the 59.38-keV (Ref. 19) experiment, denoted by triangles. The theoretical profiles are convoluted with the experimental resolution function of the $59.38-\mathrm{keV}$ experiment. The Compton profiles are in units of electrons per atom per atomic unit. 


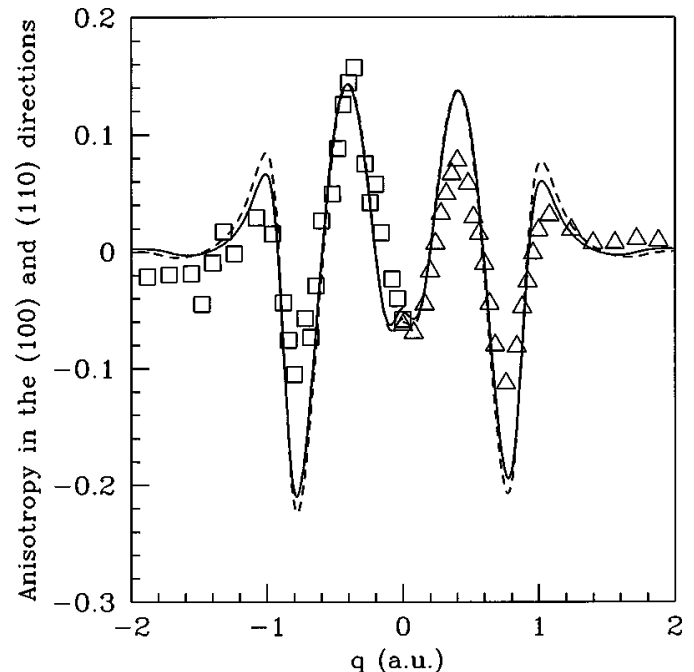

FIG. 5. The anisotropy between the (100) and (110) Compton profiles, from both the pseudopotential (dashed line) and the reconstruction (solid line) calculations, and also from experimental data at $29.5 \mathrm{keV}$ (Ref. 5) and 59.38 (Ref. 19) $\mathrm{keV}$. On the left portion, the $29.5-\mathrm{keV}$ experiment is graphed, with data points indicated by squares. On the right portion are the $59.38-\mathrm{keV}$ data, denoted by triangles. Again, the theoretical profiles are convoluted with the appropriate experimental resolution function. The anisotropies are in units of electrons per atom per atomic unit.

sity that we perform to calculate the Compton profiles, it is now much larger.

We find excellent agreement between our valence Compton profiles and those measured in the $29.5-\mathrm{keV}$ experiment, along both of the directions studied at this energy. It is clear that the reconstruction procedure goes a long way towards eliminating the discrepancy between the pseudo-wavefunction results and the experimental data. The agreement with the $59.38-\mathrm{keV}$ data is good but not as excellent, though again it is clear that the reconstruction effects bring the calculated and measured profiles appreciably closer at the origin, where the deviation is most marked. For both photon energies, the magnitude of the experimental long-range tail agrees very well with the reconstructed profiles along all of the measured directions.

Now we examine the effects of the reconstruction on the anisotropy between profiles. So, in Fig. 5 we show the anisotropy between the profiles along the (100) and (110) directions according to the pseudopotential calculation, and also the effect of reconstructing the valence wave functions. We compare with the experimental anisotropy as deduced from the $29.5-\mathrm{keV}$ and $59.38 \mathrm{-keV}$ experiments. We find good qualitative agreement between theory and experiment, though quantitatively there are differences between the heights of the peaks. First of all, we see that both theoretical calculations tend to overstate the anisotropy, especially at the largest valley near $q=0.8$ a.u. The two experiments also differ from each other, especially for the height of the peak near $q=0.4$. There also seems to be more scatter in the $29.5-\mathrm{keV}$ data. Using the reconstructed wave functions changes the theoretical curve appreciably near $q=0, q=0.8$, and $q=1$ a.u., while in other regions it changes the curve by very little.

In Fig. 6 we examine the anisotropy between the (100) and (111) directions. Again we show the pseudopotential and

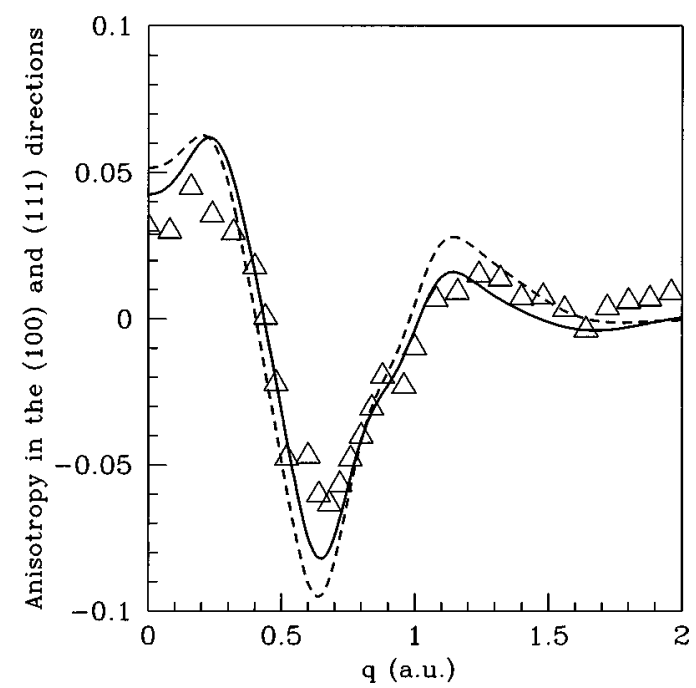

FIG. 6. The anisotropy between the convoluted (100) and (111) Compton profiles, from both the pseudopotential (dashed line) and reconstruction (solid line) calculations, and from experimental data at $59.38 \mathrm{keV}$ (Ref. 19), denoted by triangles. The anisotropies are in units of electrons per atom per atomic unit.

reconstructed curves, and compare them to the 59.38-keV experimental anisotropy. For this pair of directions, the theoretical difference profile is modified significantly by the reconstruction of the wave functions, and moves closer to the experimental values. The shift in the theoretical curve is not confined to a couple of regions, as for the (100)-(110) anisotropy, but affects the whole plot. We note that the magnitude of the maximum change in the (100)-(110) anisotropy in Fig. 5 is the same as in the Fig. 6, but because the scale of the (100)-(111) anisotropy is approximately half that of the (100)-(110) curve, the effect of reconstruction is more pronounced.

\section{CONCLUSION}

We find that the reconstruction of the all-electron valence wave functions from the pseudo-wave-functions substantially changes the valence Compton profiles of silicon along all three of the directions studied, and introduces a tail in the momentum density and profiles that persists to large momenta. Agreement with experiment is substantially improved by the use of the reconstructed wave functions, and we find excellent agreement with the $29.5-\mathrm{keV}$ data. In the anisotropy, the changes in the momentum density caused by the reconstruction procedure are not spherically symmetric, but lead to appreciable changes in the theoretical predictions, in particular for the (100)-(111) anisotropy. These changes bring the theoretical predictions closer to experiment. Because of the differences between the results from the two experiments we use for comparison, it is hard to say which of the remaining discrepancies between theory and experiment are due to experimental noise or systematic errors, and which are due to inadequacies in theory. We have also compared our theoretical results for the reconstructed valence Compton profiles with FLAPW and KKR calculations for silicon, and we find excellent agreement among all three methods. ${ }^{2}$ Elsewhere, we will examine further corrections to 
the Compton profiles due to correlation effects between the valence electrons, using a correlated wave-function quantum Monte Carlo approach.

\section{ACKNOWLEDGMENTS}

We wish to thank Bernd Meyer for giving us his reconstruction program, and also for many helpful discussions. We also thank Professor N. Shiotani and Professor Y. Sakurai for sending us their experimental data, and Professor A. Bansil for sending us a copy of the informal proceedings of the Second International Workshop on Compton Scattering and Fermiology. This work was supported by National Science Foundation Grant No. DMR-9520554 and by the Director's Office of Energy Research, Office of Basic Energy Sciences, Materials Sciences Division of the U.S. Department of Energy under Contract No. DE-AC03-76SF00098. Cray Computer time was provided by the National Science Foundation at the San Diego Supercomputer Center and at the Pittsburgh Supercomputing Center. One of us (P.D.) would like to acknowledge the support of the National University of Ireland in the first half of this work.
${ }^{1}$ Compton Scattering, edited by Brian Williams (McGraw-Hill, New York, 1976).

${ }^{2}$ Informal Proceedings of the Second International Workshop on Compton Scattering and Fermiology, August 28-31, 1995 Tokyo, Japan (unpublished).

${ }^{3}$ A. Seth and D. E. Ellis, J. Phys. C 10, 181 (1977).

${ }^{4}$ H. Nara, K. Shindo, and T. Kobayasi, J. Phys. Soc. Jpn. 46, 77 (1979).

${ }^{5}$ N. Sakai, N. Shiotani, F. Itoh, O. Mao, M. Ito, H. Kawata, Y. Ameniya, and M. Ando, J. Phys. Soc. Jpn. 58, 3270 (1989).

${ }^{6}$ Y. Sakurai, Y. Tanaka, A. Bansil, S. Kaprzyk, A. T. Stewart, Y. Nagashima, T. Hyodo, S. Nanao, H. Kawata, and N. Shiotani, Phys. Rev. Lett. 74, 2252 (1995).

${ }^{7}$ K. Hämäläinen, S. Manninen, C.-C. Kao, W. Caliebe, J. B. Hastings, A. Bansil, S. Kaprzyk, and P. M. Platzman, Phys. Rev. B 54, 5453 (1996).
${ }^{8}$ C. Blaas, J. Redinger, S. Manninen, V. Honkimäki, K. Hämäläinen, and P. Suortti, Phys. Rev. Lett. 75, 1984 (1995).

${ }^{9}$ P. Hohenberg and W. Kohn, Phys. Rev. 136, B864 (1964).

${ }^{10}$ W. Kohn and L. J. Sham, Phys. Rev. 140, A1133 (1965).

${ }^{11}$ B. Meyer, K. Hummler, C. Elsässer, and M. Fähnle, J. Phys.: Condens. Matter 7, 9201 (1995).

${ }^{12}$ D. R. Hamann, M. Schlüter, and C. Chiang, Phys. Rev. Lett. 43, 1494 (1979).

${ }^{13}$ S. G. Louie, S. Froyen, and M. L. Cohen, Phys. Rev. B 26, 1738 (1982).

${ }^{14}$ D. M. Ceperley and B. J. Alder, Phys. Rev. Lett. 45, 566 (1980).

${ }^{15}$ J. P. Perdew and A. Zunger, Phys. Rev. B 23, 5048 (1981).

${ }^{16}$ H. J. Monkhorst and J. D. Pack, Phys. Rev. B 13, 5188 (1976).

${ }^{17}$ G. Lehmann and M. Taut, Phys. Status Solidi B 54, 469 (1972).

${ }^{18}$ G. Gilat and N. R. Bharatiya, Phys. Rev. B 12, 3479 (1975).

${ }^{19}$ Y. Sakurai (private communication). 